\title{
Identifying and Evaluating Chaotic Behavior in Hydro-Meteorological Processes
}

\author{
Soojun Kim, ${ }^{1}$ Yonsoo Kim, ${ }^{2}$ Jongso Lee, ${ }^{2}$ and Hung Soo Kim ${ }^{2}$ \\ ${ }^{1}$ Columbia Water Center, Columbia University, New York, NY 10027, USA \\ ${ }^{2}$ Department of Civil Engineering, Inha University, Incheon 402-751, Republic of Korea \\ Correspondence should be addressed to Hung Soo Kim; sookim@inha.ac.kr
}

Received 20 November 2014; Accepted 7 April 2015

Academic Editor: Ismail Gultepe

Copyright (c) 2015 Soojun Kim et al. This is an open access article distributed under the Creative Commons Attribution License, which permits unrestricted use, distribution, and reproduction in any medium, provided the original work is properly cited.

\begin{abstract}
The aim of this study is to identify and evaluate chaotic behavior in hydro-meteorological processes. This study poses the two hypotheses to identify chaotic behavior of the processes. First, assume that the input data is the significant factor to provide chaotic characteristics to output data. Second, assume that the system itself is the significant factor to provide chaotic characteristics to output data. For solving this issue, hydro-meteorological time series such as precipitation, air temperature, discharge, and storage volume were collected in the Great Salt Lake and Bear River Basin, USA. The time series in the period of approximately one year were extracted from the original series using the wavelet transform. The generated time series from summation of sine functions were fitted to each series and used for investigating the hypotheses. Then artificial neural networks had been built for modeling the reservoir system and the correlation dimension was analyzed for the evaluation of chaotic behavior between inputs and outputs. From the results, we found that the chaotic characteristic of the storage volume which is output is likely a byproduct of the chaotic behavior of the reservoir system itself rather than that of the input data.
\end{abstract}

\section{Introduction}

Hydrologic phenomena arise as a result of interactions between climate inputs and landscape characteristics that occur over a wide range of space and time scales. Due to the tremendous heterogeneities in climatic inputs and landscape properties, such phenomena may be highly variable and "complex" at all scales [1]. The nonlinear behavior of hydrologic systems had been known for a long time $[2,3]$. The rainfall-runoff process is nonlinear, almost regardless of the basin area, land uses, rainfall intensity, and other influencing factors, which are changing in a highly nonlinear fashion and so are the outputs, often in unknown ways [1].

To study the nonlinear characteristics of natural phenomena, many statisticians and scientists have suggested the chaos theory which analyze and forecast the nonlinear phenomena of the natural system. Lorenz [4] suggested the strange attractor in a simple model of convection roll in the atmosphere. Packard et al. [5] suggested the method of delays and Takens [6] proved the method of delays using differential topology. Grassberger and Procaccia [7] and
Farmer et al. [8] demonstrated the estimation of chaotic characterization using correlation dimension. Wolf et al. [9] calculated the largest Lyapunov exponent using the Benettin's method. Fraser and Swinney [10] suggested a method for the estimation of time delay using the mutual information. Gilmore [11] introduced the topological method for chaos characterization, especially useful for small data sets. Farmer and Sidorowich [12] forecasted the chaotic time series using the local linear approximation. Also, Casdagli [13] forecasted the chaotic time series using the radial basis functions and Casdagli and Weigend [14] modeled and forecasted the chaotic time series using DVS (deterministic versus stochastic) algorithm. Kim et al. $[15,16]$ suggested a new method for the estimation of delay parameters in chaos analysis. Falanga and Petrosino [17] estimated the complexity of the system by the degrees of freedom necessary to describe the asymptotic dynamics in a reconstructed phase space. The mechanism of stochastic resonance, which is a nonlinear phenomenon, has been applied in the field of the physics of atmosphere since it was introduced by Benzi et al. $[18,19]$ and Nicolis [20]. 
Many hydrologists have been also analyzed hydrologic phenomena using nonlinear deterministic chaos to interpret the nonlinear characteristic of the hydrologic system. Rodriguez-Iturbe et al. [21] found the chaotic characteristics in rainfall data recorded with the time interval of 15 seconds using the correlation dimension and the Lyapunov exponent. Wilcox et al. [22] tested the chaotic behavior of daily snowmelt runoff data by correlation dimension. Sangoyomi et al. $[23,24]$ used the Great Salt Lake volume data recorded with the time interval of 15 days for searching for the chaotic characteristics. Jeong and Rao [25] used 13 tree ring series to determine their chaos characteristics. RodriguezIturbe et al. [26] investigated the nonlinear dynamics of soil moisture using a soil moisture balance equation. Kim et al. [27] searched strange attractor in wastewater flow using the C-C method. Ahn and Kim [28] showed that the nonlinear stochastic model is more valid for the SOI time series analysis and modeling than linear stochastic analog by the BDS statistic. Kim et al. [29] assessed nonlinear deterministic characteristics in hydrologic time series like rainfall, stream flow, and reservoir volume series. Sivakumar et al. [30] examined the utility of nonlinear dynamic concepts for analysis of rainfall variability across Western Australia. Kim et al. [31] assessed the applicability chaotic dynamics and filtering techniques in radar rainfall.

Even though Salas et al. [32] investigated how hydrologic process (e.g., precipitation) which is low-dimensional chaotic is changed by its transformations such as aggregation and sampling, mostly, the single hydrologic time series have been analyzed for investigating its chaotic and nonlinear dynamic characteristics. Therefore, the aim of this study is to identify chaotic behavior for the components in hydrometeorological processes such as air temperature, precipitation, discharge, and lake storage volume series. The components contribute to hydro-meteorological system as inputs and outputs. For this, the main question is, given that what is the significant factor to provide chaotic characteristics to output data? We pose the following two hypotheses. (1) Assume that the input data is the significant factor to provide chaotic characteristics to output data. (2) Assume that the system itself is the significant factor to provide chaotic characteristics to output data.

This paper is organized to solve the issue as follows. In Section 2, we give brief overview of the methodology to estimate the correlation dimension, which can detect chaotic characteristics of data series. In Section 3, we also give brief overview of the wavelet transform to extract the data of the representative period from the original time series and artificial neural networks (ANN) for modeling the hydrometeorological system. In Section 4, we apply methods for identifying chaotic behavior of the data series and discuss the results. Finally, in Section 5, we summarize the findings and conclusions.

\section{Estimation of Correlation Dimension}

2.1. Phase Space Reconstruction. Phase space is a useful tool for representing the evolution of a system in time.
It is essentially a graph or a coordinate diagram, whose coordinates represent the variables necessary to completely describe the state of the system at any moment (in other words, the variables that enter the mathematical formulation of the system). The trajectories of the phase space diagram describe the evolution of the system from some initial state, which is assumed to be known, and, hence, represent the history of the system [5]. The "region of attraction" of these trajectories in the phase space provides at least important qualitative information on the "extent of complexity" of the system, which can subsequently be verified quantitatively using methods based on, for example, the concept of dimensionality.

For a dynamic system with known partial differential equations (PDEs), the system can be studied by discretizing the PDEs, and the set of variables at all grid points constitutes a phase space. One difficulty in constructing the phase space for such a system is that the (initial) values of many of the variables may not be known. However, a time series of a single variable of the system may be available, which may allow the attractor (a geometric object that characterizes the long-term behavior of a system in the phase space) to be reconstructed. The idea behind such a reconstruction is that a (nonlinear) system is characterized by self-interaction, so that a time series of a single variable can carry the information about the dynamics of the entire multivariable system. Many methods are available for phase space reconstruction from an available time series. Among these, the method of delays (e.g., [6]) is the most widely used one. According to this method, given a single-variable series, $X_{i}$, where $i=1,2, \ldots, N$, a multidimensional phase space can be reconstructed as

$$
Y_{j}=\left(X_{j}, X_{j+\tau}, X_{j+2 \tau}, \ldots, X_{j+(m-1) \tau}\right)
$$

where $j=1,2, \ldots, N-(m-1) \tau ; m$ is the dimension of the vector $Y_{j}$, called embedding dimension; and $\tau$ is an appropriate delay time (an integer multiple of sampling time). A correct phase space reconstruction in a dimension $m$ generally allows interpretation of the system dynamics (if the variable chosen to represent the system is appropriate) in the form of an $m$-dimensional map $f_{T}$, given by

$$
Y_{j+T}=f_{T}\left(Y_{j}\right)
$$

where $Y_{j}$ and $Y_{j+T}$ are vectors of dimension $m$, describing the state of the system at times $j$ (current state) and $j+T$ (future state), respectively.

2.2. Correlation Integral and Correlation Dimension. The dimension of a time series is, in a way, a representation of the number of variables dominantly governing the underlying system dynamics. Correlation dimension is a measure of the extent to which the presence of a data point affects the position of the other points lying on the attractor in the phase space. The correlation dimension method uses the correlation integral (or function) for determining the dimension of the attractor and, hence, for distinguishing between low-dimensional chaos and high-dimensional system. The concept of the correlation integral is that a time 
series arising from deterministic dynamics will have a limited number of degrees of freedom equal to the smallest number of first-order differential equations that capture the dominant features of the dynamics. Thus, when one constructs phase spaces of increasing dimension, a point will be reached where the dimension equals the number of degrees of freedom, beyond which increasing the phase space dimension will not have any significant effect on correlation dimension. Many algorithms have been formulated for the estimation of the correlation dimension. Among these, the GrassbergerProcaccia algorithm [7] has been the most popular. The algorithm uses the concept of phase space reconstruction for representing the dynamics of the system from an available single-variable time series, as presented in (1). For an mdimensional phase space, the correlation integral or function $C(r)$ is given by

$$
C(r)=\lim _{N \rightarrow \infty} \frac{2}{N(N-1)} \sum_{i j} H\left(r-\left|Y_{i}-Y_{j}\right|\right),
$$

where $H$ is the Heaviside step function, with $H(u)=1$ for $u>0$ and $H(u)=1$ for $u \leq 0$, where $u=r-\left\|Y_{i}-Y_{j}\right\|, r$ is the vector norm (radius of sphere) centered on $Y_{i}$ or $Y_{j}$. If the time series is characterized by an attractor, then $C(r)$ and $r$ are related according to

$$
C(r)_{r \rightarrow 0, N \rightarrow \infty} \approx \alpha r^{\nu}
$$

where $\alpha$ is a constant and $\nu$ is the correlation exponent or the slope of the $\log C(r)$ versus $\log r$ plot. The slope is generally estimated by a least square fit of a straight line over a certain range of $r$ (scaling regime) or through estimation of local slopes between $r$ values. The distinction between lowdimensional (perhaps determinism) and high-dimensional (perhaps stochasticity) can be made using the $v$ versus $m$ plot. If $v$ saturates after a certain $m$ and the saturation value is low, then the system is generally considered to exhibit low-dimensional and possibly deterministic dynamics. The saturation value of $v$ is defined as the correlation dimension (D2) of the attractor, and the nearest integer above this value is generally an indication of the number of variables dominantly governing the dynamics. On the other hand, if $m$ increases without bound with increase in $m$, the system under investigation is generally considered to exhibit highdimensional and possibly stochastic behavior.

\section{Wavelet Transform and Artificial Neural Networks}

3.1. Wavelet Transform. According to Fourier theory, a signal can be expressed as the sum of a possibly infinite series of sine and cosines, referred to as a Fourier expansion [33]. However, a Fourier expansion has only frequency resolution and not time resolution; that is, no amplitude modulation of the signal at a given frequency is considered. Movingwindow Fourier transforms have been used to address this issue, but this method is sensitive to the choice of window width. Alternatively, the wavelet transform [34, 35] enables the identification of frequency components as well as their variation in time. The continuous wavelet transform of a discrete sequence $x n$ is defined by the convolution of $x_{n}$ with a scaled and translated wavelet function $\psi$ :

$$
W_{n}(s)=\sum_{n^{\prime}=0}^{N-1} x_{n^{\prime}} \psi *\left[\frac{\left(n^{\prime}-n\right) \delta t}{s}\right]
$$

where $(*)$ indicates the complex conjugate, $n$ is the localized time index, $s \neq 0$ is the scale parameter, and $N$ is the number of points in the time series. In this study, we use the Morlet wavelet function defined as $\psi(\eta)=\pi^{-1 / 4} e^{i \omega_{0} \eta} e^{\eta^{2} / 2}$, where $\omega_{0}$ is a frequency and $\eta$ is a nondimensional "time" parameter. By varying the wavelet scales and translating along the localized time index $n$, one can construct a picture that shows both the amplitude of any features versus the scale and how this amplitude varies with time. A vertical slice through a wavelet plot is a measure of the local spectrum. The time-averaged wavelet spectrum over all the local wavelet spectra gives the global wavelet spectrum:

$$
\overline{W_{t}^{2}}(s)=\frac{1}{T} \sum_{t=0}^{T-1}\left|W_{t}(s)\right|^{2}
$$

A more detailed presentation for wavelet transform analysis is referred to read Torrence and Compo [35].

3.2. Artificial Neural Networks. ANN is a model of neurotransmission by a neuron, which is a nerve cell in the human brain. ANN is an empirical pattern search technique that enables the consideration of a nonlinear relationship between input variables and output variables. ANN is used in various areas because of its unique applicability $[36,37]$. This includes the field of climate science, where its applicability is proven $[38,39]$.

Many studies suggest the ANN technique, which is a nonlinear model of the data series, and ANN is better than other techniques by way of systematic evaluation of various techniques $[40,41]$. Therefore, this study also applies ANN, which is judged to have superior applicability in the simulation of nonlinear characteristics of the hydro-meteorological system.

\section{Applications and Results}

4.1. Study Area and Data Series Used. The Bear River Basin, located in northeastern Utah, southeastern Idaho and southwestern Wyoming, comprises 7,500 square miles of mountain and valley lands including 2,700 in Idaho, 3,300 in Utah, and 1,500 in Wyoming. The Bear River crosses state boundaries five times and is the largest stream in the western hemisphere that does not empty into the ocean. It ranges in elevation from over 1,278 to 3,868 feet and is unique in that it entirely enclosed by mountains, thus forming a huge basin with no external drainage outlets (http://www.greatsaltlakeinfo.org/Background/BearRiver). The Bear River is the largest tributary to the Great Salt Lake (see Figure 1). 


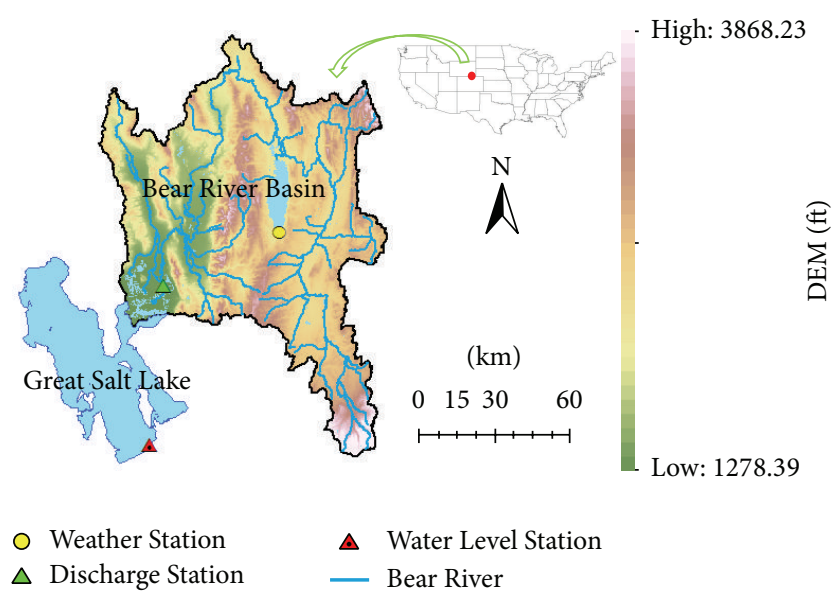

Figure 1: Study area.

The data was collected from weather gauging station (USC00424856, NOAA), stream flow gauging station (number 10126000, USGS), and lake water level gauging station (number 10010000, USGS) for the period of 1903 to 1995. The monthly rainfall shows its statistics of average $258.5 \mathrm{~mm}$ and standard deviation $216.4 \mathrm{~mm}$, the monthly mean temperature shows average $5.7^{\circ} \mathrm{C}$ and standard deviation $8.8^{\circ} \mathrm{C}$, the monthly mean runoff shows average $1533.6 \mathrm{ft}^{3} / \mathrm{s}$ and standard deviation $1331.2 \mathrm{ft}^{3} / \mathrm{s}$, the monthly mean storage in the lake shows average $149.4 \times 10^{5} \mathrm{ft}^{3}$ and standard deviation $39.8 \times$ $10^{5} \mathrm{ft}^{3}$, and these time series plots are shown in Figure 2.

\subsection{Extraction of a Representative Time Series by Wavelet} Transform. All hydrological measurements are to some extent contaminated by noise. And the noise limits the performance of many techniques of identification, modeling, prediction, and control of deterministic systems [42]. Independent component analysis (ICA) as a popular method is able to extract periodic signals from noise or nonlinear mixture $[43,44]$. It has been applied in the fields of meteorology [45], oceanography [46], volcanology [47, 48], and remote sensing [49]. This study, however, uses wavelet transform for extracting the representative periodic components which affect the data series because ICA often leads to local minimum solution and the suitable source signals are not isolated [50]. Moreover, the order of the independent components (ICs) is difficult to be determined in comparison with wavelet transform.

Wavelet power spectrum that estimated the wavelet mother function using the Morlet function is shown in Figure 3 (left) and the extent of spectrum in each period for time series can be identified. In this figure, a solid halfcircle line shows the edge of the cone of influence (COI) effect that can be caused by the discontinuity of the beginning and end of data series. In particular, the upper part of the solid line is statistically significant (a 95\% confidence interval) and the lower part is excluded from interpretation. Parts with high-density spectrum are observed in some periods within a confidence interval. Global wavelet power spectrum (GWP) in Formula (6), which represents the average value according to the length of each period, provides more effective information about spectrum. Figure 3 (center) shows the result of GWP about spectrum. Considering that the right part of a solid line is statistically significant on a basis of a $95 \%$ confidence level, the periodic characteristics of the time series could be classified into one band. The band shows a strong spectrum of the period of approximately 1 year. The period extracted from the wavelet spectrum is shown in the right of Figure 3.

\subsection{Analysis of the Time Series Using Attractor and Correlation Dimension}

4.3.1. Attractor Analysis. The attractor obtained by (1) can describe the characteristics of a time series. To obtain the attractor using (1), the index lag $t$ and embedding dimension $m$ must be chosen appropriately. The autocorrelation function (ACF) is expected to provide a reasonable measure of the transition from redundance to irrelevance as a function of delay. The decorrelation time which is equal to the lag (delay time: $\tau$ ) at which the ACF first attains the value zero is considered. Otherwise, $\tau$ should be chosen as the local minimum of ACF, whichever occurs first $[51,52]$. When the ACF decays exponentially, we select $\tau$ at which the ACF drops to zero [53], at lag time 4 months in all of series. Therefore, the delay times of the systems can be obtained from ACFs and the attractors are drawn in Figure 4 for each time series.

For the attractor analysis, this study uses the extracted time series by the wavelet transform. The attractors of the time series have a circle with a boundary. If the attractor in the phase-space exhibits clearly within a very well defined boundary, suggest that the dynamics are simple and the system is potentially low dimension. Every time series have has a shape with a boundary which looks like a chaotic series. Particularly air temperature shows a very well defined boundary, and it is potentially low-dimensional series. Precipitation, however, shows relatively high complex and irregular, and it is a potentially high-dimensional system than the other data series.

4.3.2. Correlation Dimension Analysis. Figure 5 shows the relationship between the correlation dimension, D2, and the embedding dimensions, $m$, from 1 to 15 , for each time series. The correlation dimension seems to increase with the embedding dimension up to a certain point and saturate beyond that point. Such a saturation of the correlation dimension is an indication of the existence of deterministic dynamics. The saturation values of the correlation dimension for the series are showing 3.92, 1.41, 3.02, and 2.65 in Figures $5(\mathrm{a})-5(\mathrm{~d})$. The low correlation dimensions suggest the presence of low-dimensional chaotic nature of the underlying system dynamics. As the nearest integer above the correlation dimension value generally provides the number of dominant variables influencing the dynamics of the underlying system, the correlation dimensions for the series indicate that the time series of precipitation, air temperature, discharge, and storage volume are dominantly governed by four, two, four, 


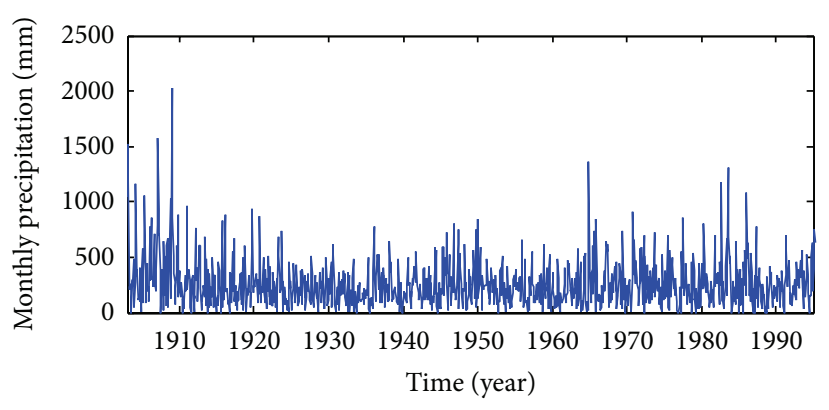

(a) Precipitation

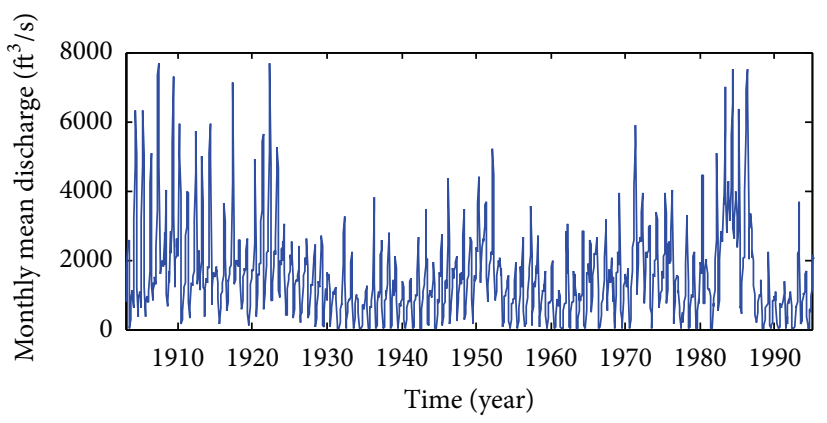

(c) Discharge

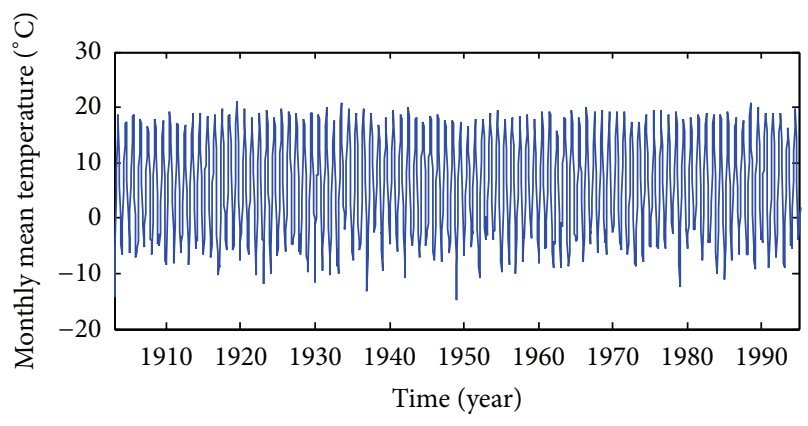

(b) Air temperature

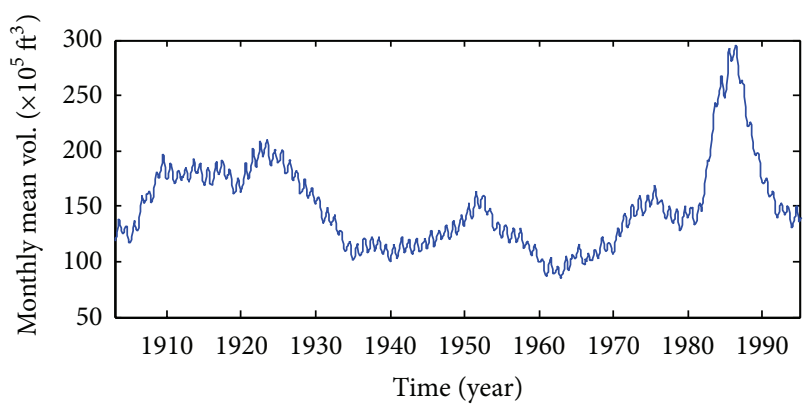

(d) Storage volume

FIgURE 2: Monthly time series plots for the period of 1903-1995.

and three variables, respectively. Here we can find the truth that the time series have different chaos characteristics even if they are collected from a hydro-meteorological system.

\subsection{Correlation Dimension Analysis Using Synthetically Gen-} erated Series. Precipitation and air temperature from the meteorological system are considered as input time series of the runoff system. On the same principle, the output series which is a discharge at the runoff system or the basin outlet occurred by input series of precipitation and air temperature from the meteorological system can be the input data of the reservoir system. Here the methodology is suggested to solve the two hypotheses as follows. We composed the input data sets, which have an arbitrary correlation dimension and build up ANNs as a nonlinear model for modeling the reservoir system. The modeling results from the input data sets will be the criterion of the hypotheses. The first hypothesis will be reasonable if the system responses sensitively depending on the arbitrary input data sets, whereas the second hypothesis will be reasonable if the system does not response sensitively depending on the input data sets.

\subsubsection{Correlation Dimensions of Generated Input Series to the} Reservoir System. The attractors in each time series (shown in Figure 4) have limit cycle regime which is the characteristics of a periodic system. Each time series as a periodic function can be written as an infinite sum of sine and cosine terms. Fourier [54] realized this first, so that this infinite sum is called a Fourier series.
The input data sets are composed of the nine sets using the three sine functions in the each hydro-meteorological time series. Here the sine function, which is useful for application to a periodic time series data, is made using the fitting toolbox of MATLAB. Therefore each time series is composed of the three cases of case (a), case (b), and case (c) as shown in Table 1. Case (a) is composed of the sum of few sine functions and case (c) is composed of the sum of lots of sine functions relatively. Case (b) is between case (a) and case (c). In case of precipitation and discharge, the functions are set to have at least three sine functions because the series are dominantly governed by four variables from the results of the correlation dimension analysis in Section 4.3.2. It is found that the fitting results have a good applicability, with the correlation coefficient (CC) in precipitation $0.54-0.65$, air temperature $0.98-0.99$, and discharge $0.88-0.92$ for 1116 months (19031995). The results of the correlation dimension analysis in each case are shown in Figure 6. The saturated correlation dimensions in each series are (a) 2.54, (b) 3.26, and (c) 4.05 in precipitation, (a) 1.02, (b) 1.84, and (c) 2.52 in air temperature, and (a) 2.48, (b) 3.13, and (c) 3.8. Case (c) is composed of many sine functions which showed the highest correlation dimension, whereas case (a) shows the lowest correlation dimension in each time series.

4.4.2. ANN Modeling and Correlation Dimension Analysis of Hydro-Meteorological System. In order to build up the ANN model, this study sets precipitation, air temperature, and discharge as the input layer and storage volume as the output layer. As seen in Figure 7, a multilayered ANN model 
TABle 1: Fitting functions in each case of each time series.

\begin{tabular}{lll}
\hline & Fitting function & CC \\
\hline \multirow{4}{*}{ Precipitation } & $(\mathrm{a})=1.51 \cdot \sin (0.53 \cdot t-0.50)+15.5 \cdot \sin (0.54 \cdot t-1.94)+15.48 \cdot \sin (0.54 \cdot t+1.02)$ & 0.54 \\
& $(\mathrm{~b})=1.50 \cdot \sin (0.53 \cdot t-0.53)+18.81 \cdot \sin (0.54 \cdot t-2.02)+18.82 \cdot \sin (0.54 \cdot t+0.97)+1.23 \cdot \sin (0.55 \cdot t-0.61)$ & 0.60 \\
& $(\mathrm{c})=1.50 \cdot \sin (0.53 \cdot t-0.53)+19.49 \cdot \sin (0.54 \cdot t-2.02)+19.5 \cdot \sin (0.54 \cdot t+0.98)+1.23 \cdot \sin (0.55 \cdot t-0.60)+$ & 0.65 \\
& $1.131 \cdot \sin (0.50 \cdot t+0.63)$ & 0.98 \\
\hline \multirow{2}{*}{ Air } & $(\mathrm{a})=16.15 \cdot \sin (0.52 \cdot t-2.21)$ & 0.99 \\
temperature & $(\mathrm{b})=16.17 \cdot \sin (0.52 \cdot t-2.22)+0.38 \cdot \sin (0.51 \cdot t+0.46)$ & 0.99 \\
\hline & $(\mathrm{c})=16.14 \cdot \sin (0.52 \cdot t-2.20)+0.37 \cdot \sin (0.51 \cdot t+0.44)+0.33 \cdot \sin (0.53 \cdot t-5.16)$ & 0.88 \\
Discharge & $(\mathrm{a})=7.68 \cdot \sin (0.52 \cdot t-0.59)+12.02 \cdot \sin (0.53 \cdot t-2.61)+11.11 \cdot \sin (0.53 \cdot t+0.19)$ & 0.89 \\
& $(\mathrm{~b})=7.66 \cdot \sin (0.52 \cdot t-0.57)+12.86 \cdot \sin (0.53 \cdot t-2.69)+12 \cdot \sin (0.53 \cdot t+0.15)+1.08 \cdot \sin (0.55 \cdot t-2.43)$ & \\
& $(\mathrm{c})=37.23 \cdot \sin (0.52 \cdot t-1.68)+31.61 \cdot \sin (0.52 \cdot t+1.16)+0.72 \cdot \sin (0.53 \cdot t-1.67)+0.98 \cdot \sin (0.55 \cdot t-2.51)+$ & 0.92 \\
\hline & $1.20 \cdot \sin (0.51 \cdot t-1.77)$ & \\
\hline
\end{tabular}
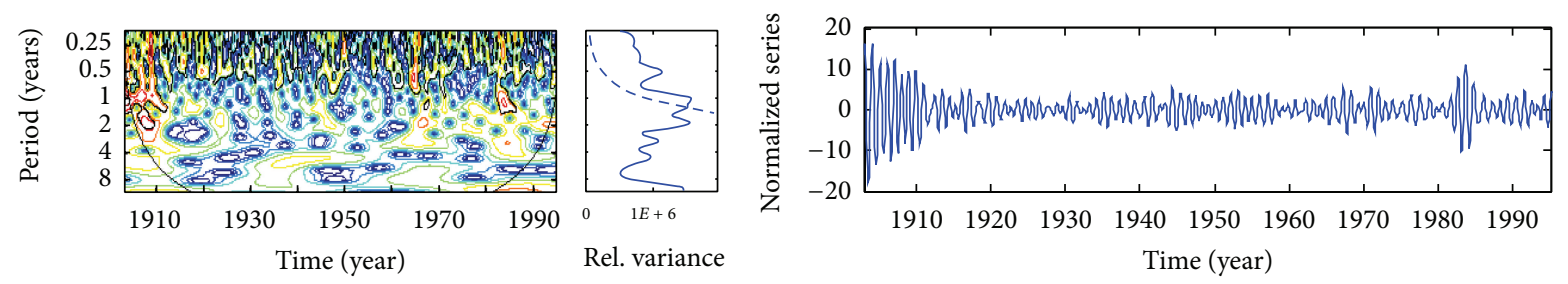

(a) Precipitation
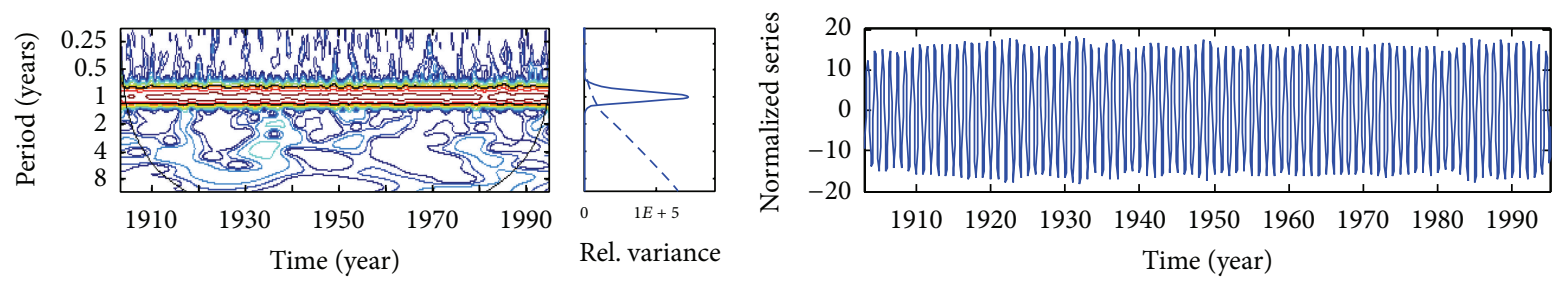

(b) Air temperature
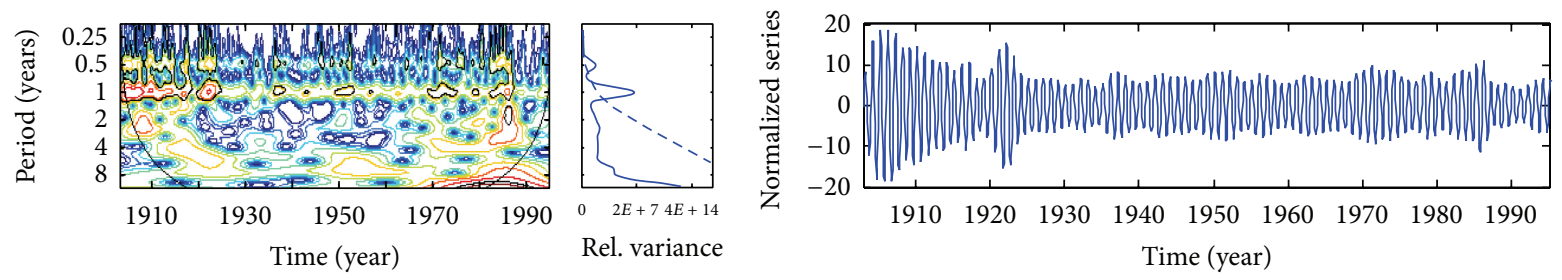

(c) Discharge
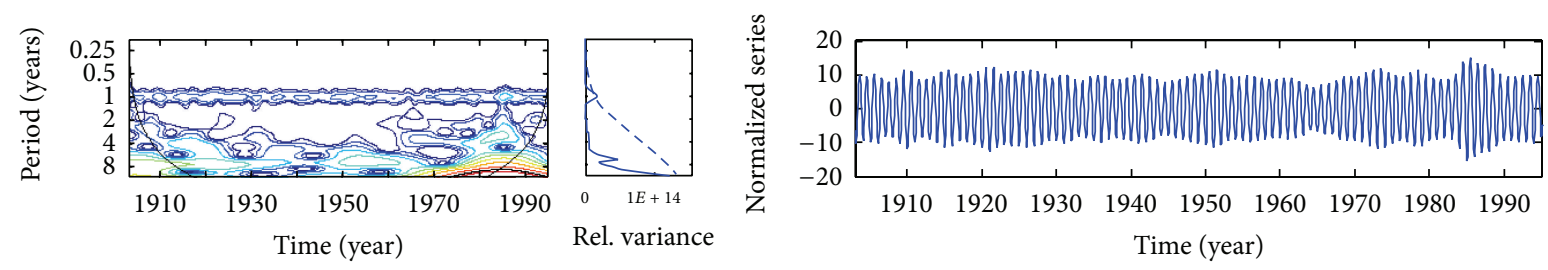

(d) Storage volume

FIGURE 3: Extraction of the representative time series using the wavelet transform (left: the wavelet power spectrum, center: the global wavelet power spectrum, and right: the extracted time series about the period of approximately 1 year).

consisting of one input layer, two hidden layers, and one output layer has been built.

Monthly data series from 1903 to 1970 (800 months) has been used for the learning period. 316 months from the learning period (1970-1995) are set up as verification periods and the applicability of the constructed ANN model is reviewed by comparing it to observed storage volume as a target data series (see Table 2). For the composition of the prediction data, we again compose the three input data like cases (A), (B), and (C). Case (A) is integrated from each 


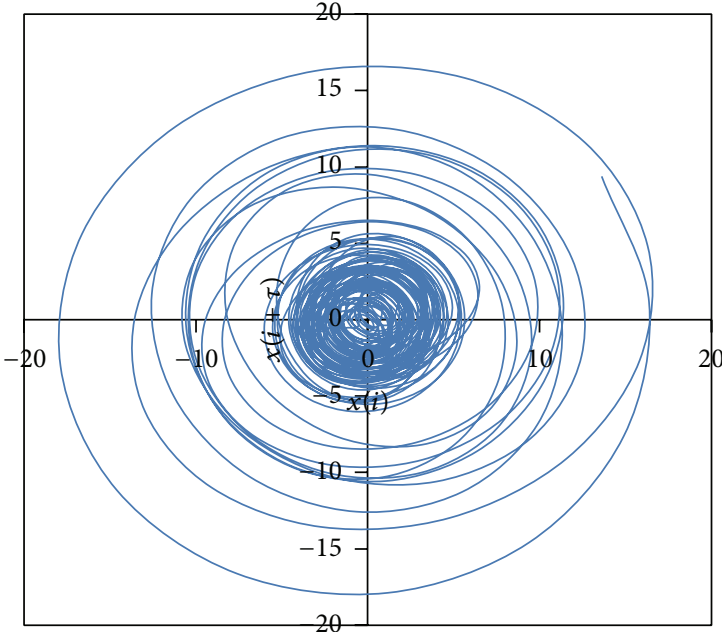

(a) Precipitation

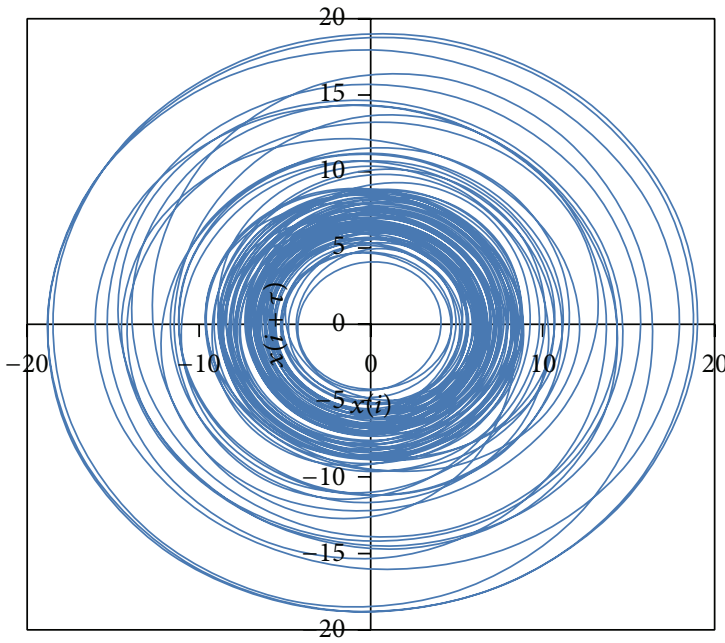

(c) Discharge

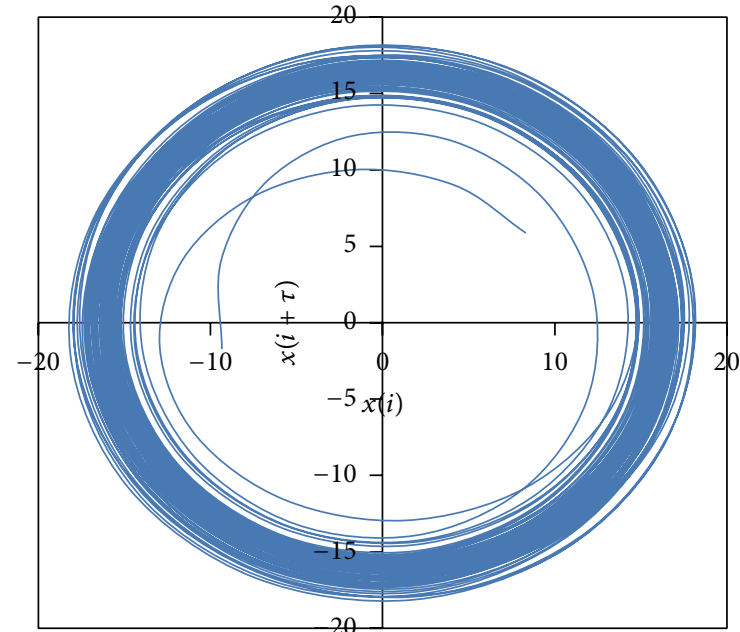

(b) Air temperature

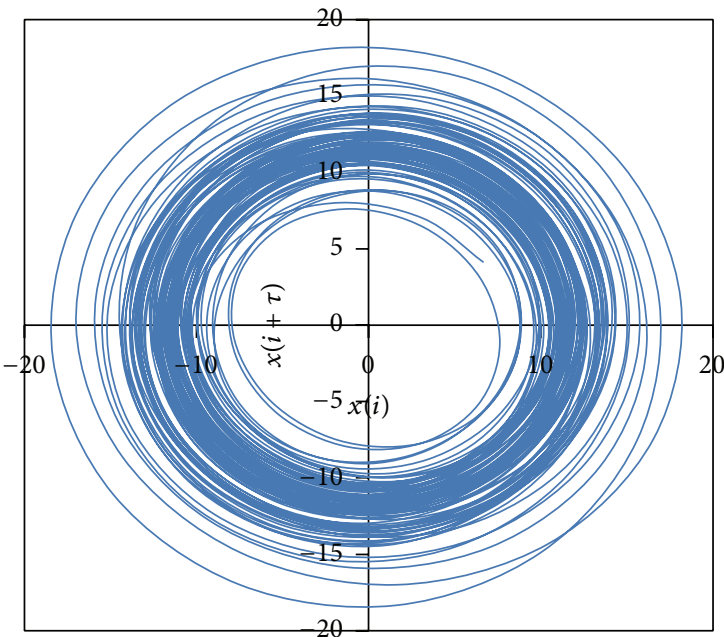

(d) Storage volume

Figure 4: Attractors in each time series.

case (a) in each time series. Case (B) and case (C) are also integrated in the same way as case (A). Data series of 1116 months (1903-1995) in each case (A), (B), and (C) has been used for the prediction period.

First of all, according to the model verification measures (see Figure 8), such as the coefficient of correlation (CC, 0.986 ) and root mean squared error (RMSE, 0.061), ANN is fitted very well and found its good applicability.

The storage volume series of a reservoir system is estimated using the ANN model after setting case (A), case (b), and case (c) as the input data. And then the correlation dimension analysis is performed for the estimated storage volume in each case. The results show that 2.55 in case (A) integrated the low-dimensional cases (a), 2.81 in case (B) integrated the middle-dimensional cases (b), and 2.89 in case (C) integrated the high-dimensional cases (c) as shown in Figure 9.
4.5. Summary and Discussions. In this study, we posed the two hypotheses to identify chaotic behavior in hydrometeorological processes. For solving this issue, we composed the input data sets like cases (A), (B), and (C) and applied them to ANN model on the reservoir system of the Great Salt Lake. The criterion of the hypotheses is the sensitivity of chaotic behavior in the system. In other words, the first hypothesis is reasonable if chaotic behavior in the system is sensitive depending on chaotic characteristics of the input data; otherwise, the second hypothesis is reasonable. The results of the correlation dimension analysis on every case analyzed in this study were summarized in Table 3.

As shown in Table 3, the correlation dimensions are 2.55 in case (A) obtained from integrating the low dimensions (precipitation 2.54, air temperature 1.02, and discharge 2.48) and 2.81 in case (B) from integrating the middle dimensions (precipitation 3.26, air temperature 1.84, and discharge 3.31) 
TABLE 2: Input data of ANN.

\begin{tabular}{lcc}
\hline & Test & Prediction \\
\hline & Precipitation & Case (A) \\
Data & Air temperature & Case (B) \\
& Discharge & Case (C) \\
Learning (calibration) period & Storage volume & - \\
& $1903 \sim 1970$ (68 years) & - \\
Verification period & $1-800(800$ months $)$ & - \\
Prediction period & $1970 \sim 1995(26$ years $)$ & $1-1116$ (1116 months) \\
\hline
\end{tabular}

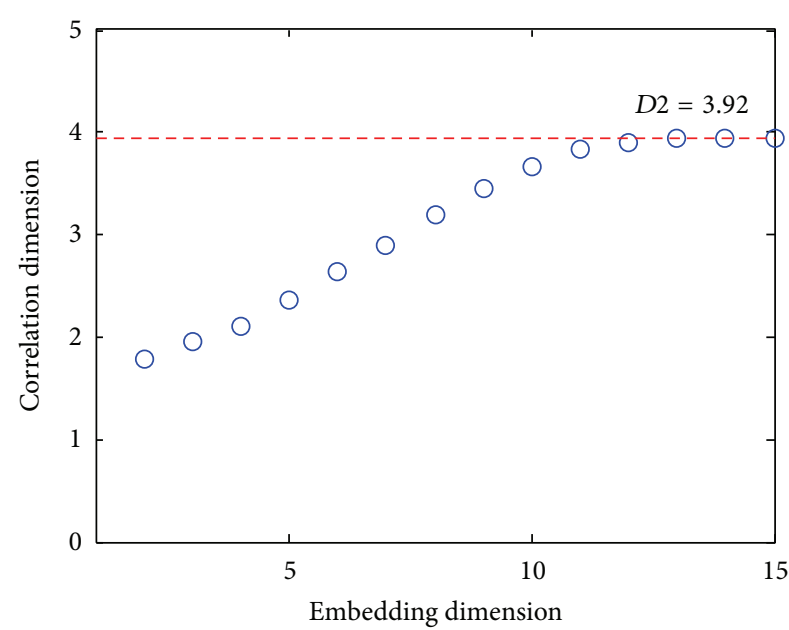

(a) Precipitation

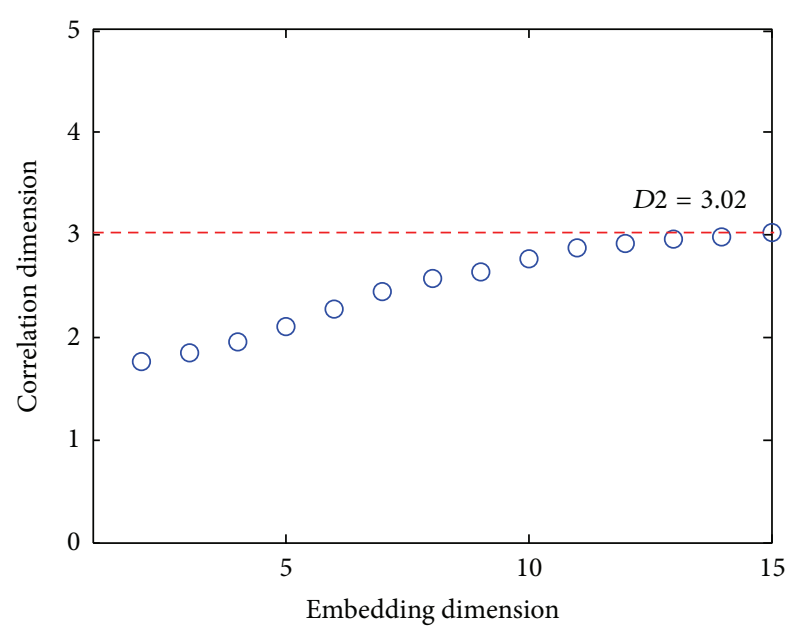

(c) Discharge

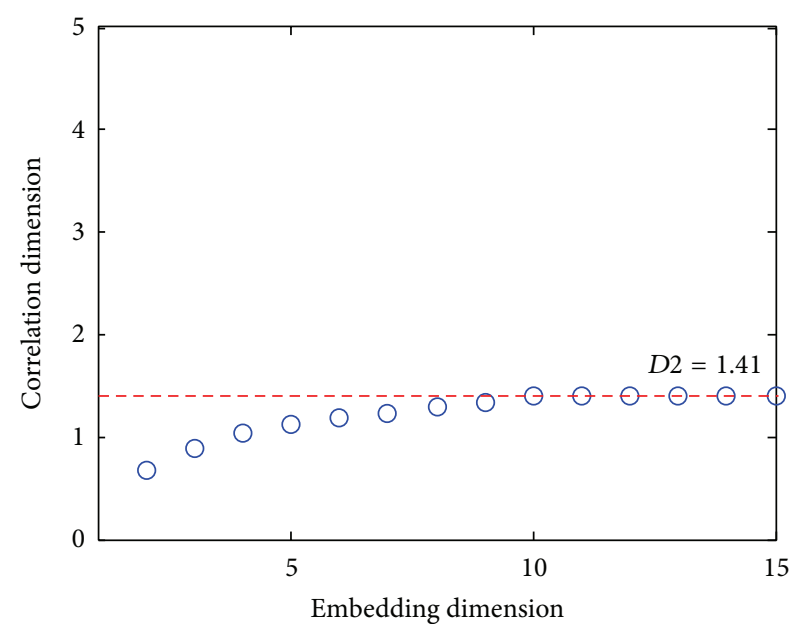

(b) Air temperature

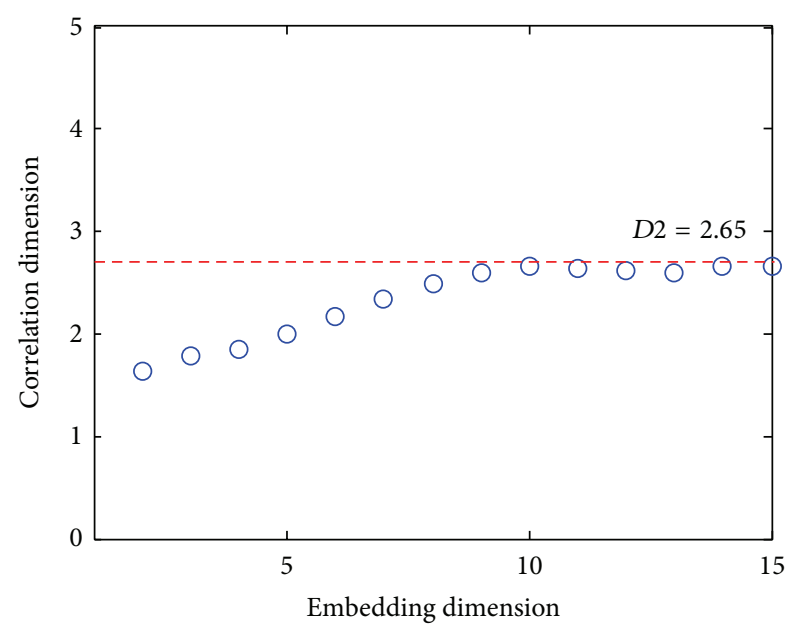

(d) Storage volume

FIgURE 5: The estimated correlation dimension for each time series.

and 2.89 in case (C) from integrating the highest dimensions (precipitation 4.05, air temperature 2.52, and discharge 3.80). The input data did not impact significantly on chaotic characteristics of the storage volume as the output even though there was a little difference of the dimension 0.34 between case (A) and case (B). Therefore the chaotic characteristic of the storage volume output in the Great Salt Lake is most likely a byproduct of the chaotic behavior of the reservoir system itself rather than that of the input data. However this chaotic behavior will depend on each hydro-meteorological system. 

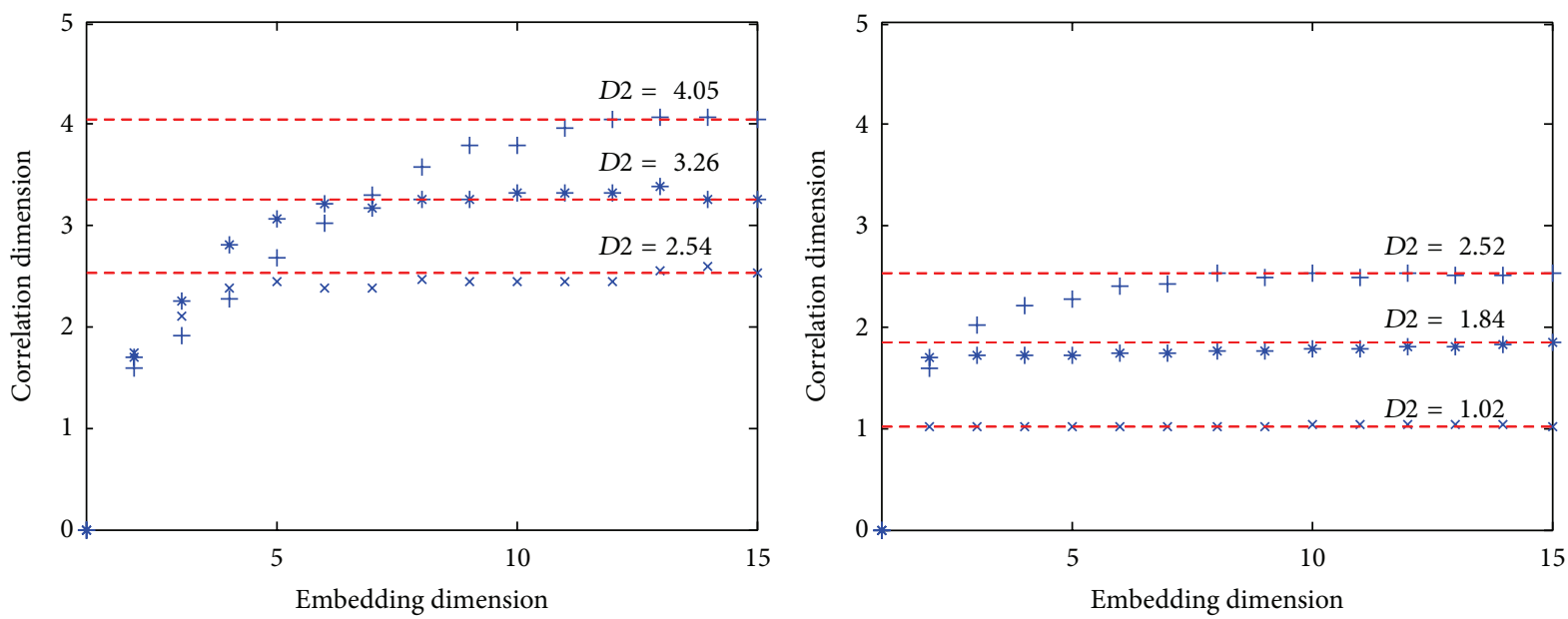

$\times(\mathrm{a})$

* (b)

$\times($ a)

* (b)

$+(\mathrm{c})$

(i) Precipitation

(ii) Air temperature

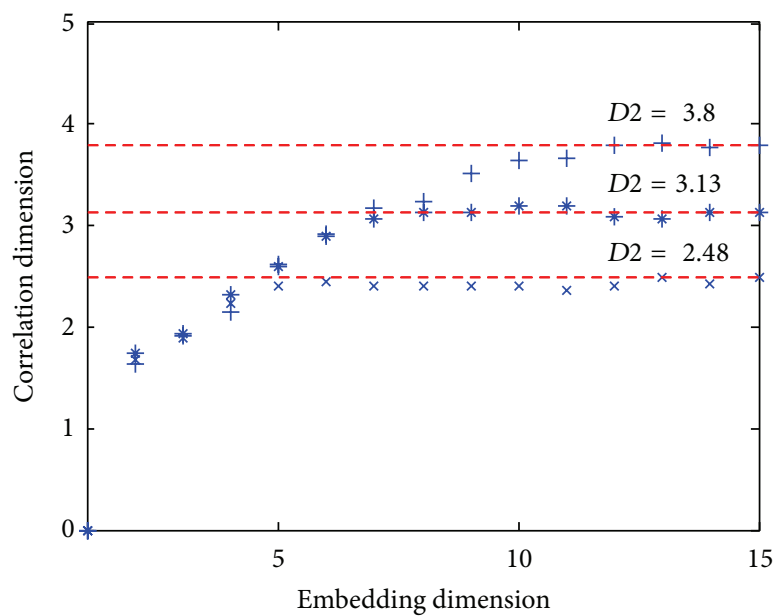

$\times($ a)

* (b)

$+(\mathrm{c})$

(iii) Discharge

FIgURE 6: Correlation dimensions of generated time series.

TABLE 3: Summary of correlation dimension in each case and time series.

Correlation dimension $(D 2)$

Precipitation Air temperature Discharge GSL volume

\begin{tabular}{lcccc}
\hline Case (A) & (a) 2.54 & (a) 1.02 & (a) 2.48 & 2.55 \\
Case (B) & (b) 3.26 & (b) 1.84 & (b) 3.13 & 2.81 \\
Case (C) & (c) 4.05 & (c) 2.52 & (c) 3.80 & 2.89 \\
Initial data & 3.92 & 1.41 & 3.02 & 2.65
\end{tabular}

For example, small hydro-meteorological systems will be very sensitive and the chaotic characteristic will be also sensitive depending on the input data.

\section{Conclusions}

This study tried to identify and evaluate chaotic behavior in hydro-meteorological processes. For solving the issue suggested in this study, the two hypotheses were posed. First, assume that the input data is the significant factor to provide chaotic characteristics to output data. Second, assume that the system itself is the significant factor to provide chaotic characteristics to output data. The hydro-meteorological time series such as precipitation, air temperature, discharge, and storage volume were collected in the Great Salt Lake and Bear River Basin and the time series in the period of approximately one year were extracted from the original time series using the wavelet transform. The results of the correlation dimension analysis showed precipitation 3.92, air temperature 1.41, 


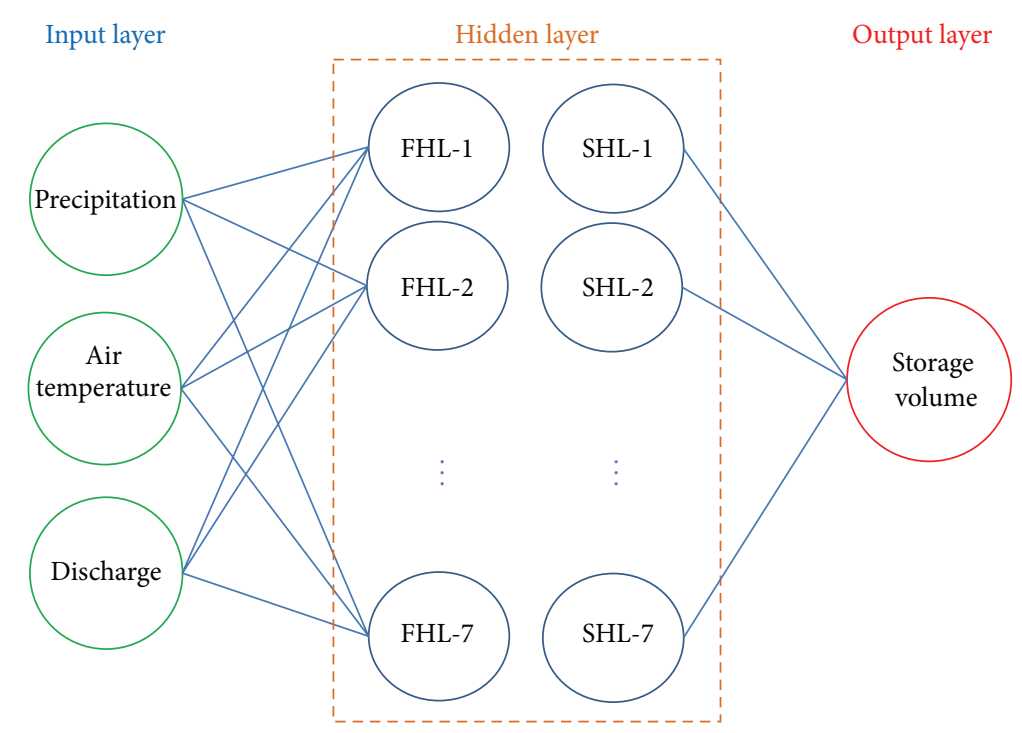

FIGURE 7: Building up ANN model.

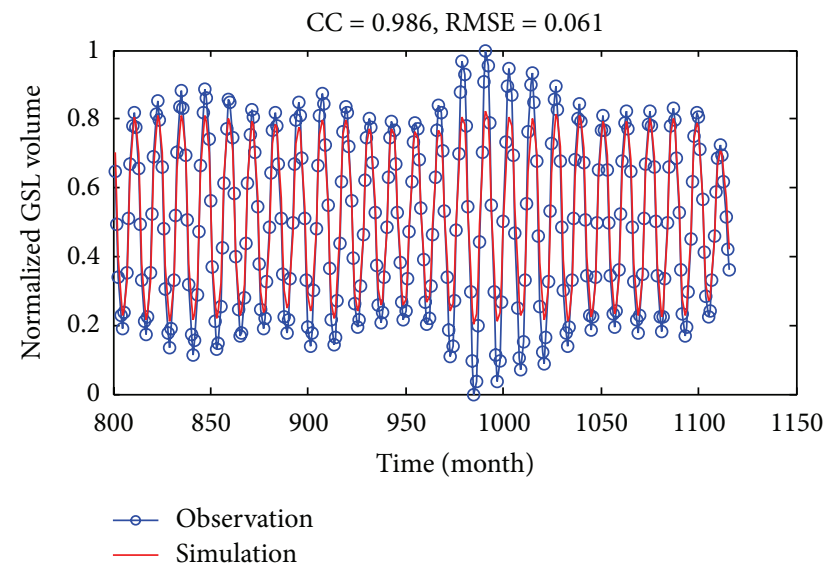

FIGURE 8: Verification result of ANN model for 316 months (19701995).

discharge 3.02, and storage volume 2.65 in each time series. The input data sets by the summation of sine functions were composed and applied them to the artificial neural networks for modeling the reservoir system depending on the data sets and integrated the high, middle, and low dimensions. Finally the correlation dimension was analyzed to evaluate chaotic behavior of storage volume which is the final output with inputs of precipitation, air temperature, and discharge in the hydro-meteorological system. The results showed that the chaotic characteristic of the storage volume is most likely a byproduct of the chaotic behavior of the reservoir system itself rather than that of the input data. We expect that the methodology and procedure suggested in this study will provide a clue to understand chaotic behavior in hydrometeorological processes.

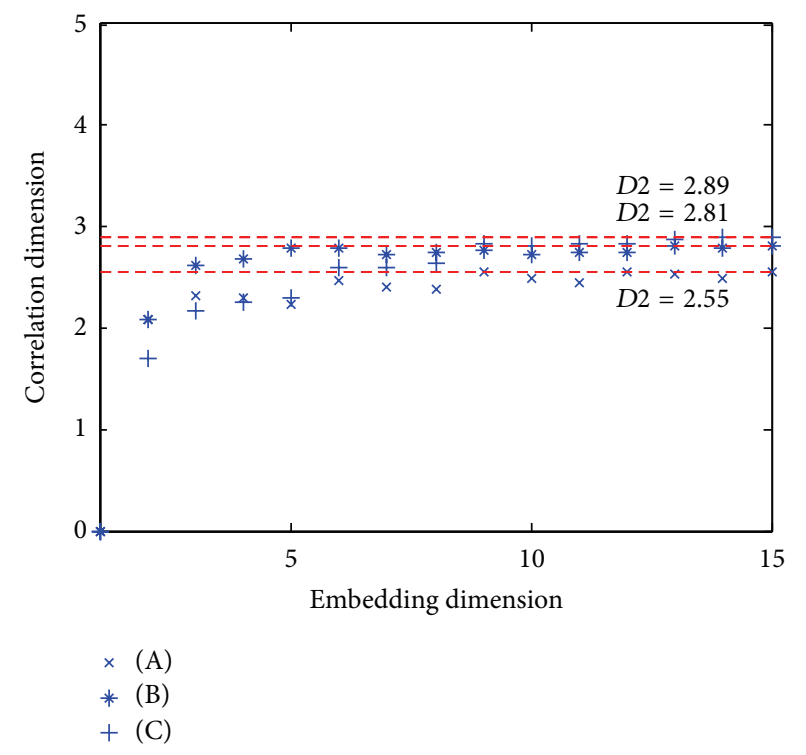

Figure 9: Correlation dimension results in each case (A), (B), and (C).

\section{Conflict of Interests}

The authors declare that there is no conflict of interests regarding the publication of this paper.

\section{Acknowledgments}

This work was supported by the National Research Foundation of Korea (NRF) and grant funded by the Korean Government (MEST; no. 2011-0028564). Also, this work was supported by INHA UNIVERSITY Research Grant. 


\section{References}

[1] B. Sivakumar and V. P. Singh, "Hydrologic system complexity and nonlinear dynamic concepts for a catchment classification framework," Hydrology and Earth System Sciences, vol. 16, no. 11, pp. 4119-4131, 2012.

[2] S. L. S. Jacoby, "A mathematical model for nonlinear hydrologic systems," Journal of Geophysical Research, vol. 71, no. 20, pp. 4811-4824, 1966.

[3] J. C. I. Dooge, "A new approach to nonlinear problems in surface water hydrology: hydrologic systems with uniform nonlinearity," The International Association of Hydrological Sciences, vol. 76, pp. 409-413, 1967.

[4] E. N. Lorenz, "Deterministic nonperiodic flow," Journal of the Atmospheric Sciences, vol. 20, no. 2, pp. 130-141, 1963.

[5] N. H. Packard, J. P. Crutchfield, J. D. Farmer, and R. S. Shaw, "Geometry from a time series," Physical Review Letters, vol. 45, no. 9, pp. 712-716, 1980.

[6] F. Takens, "Detecting strange attractors in turbulence," in Dynamical Systems and Turbulence, D. A. Rand and D. A. Young, Eds., vol. 898 of Lecture Notes in Mathematics, pp. 336-381, Springer, Berlin, Germany, 1981.

[7] P. Grassberger and I. Procaccia, "Measuring the strangeness of strange attractors," Physica D: Nonlinear Phenomena, vol. 9, no. 1-2, pp. 189-208, 1983.

[8] J. D. Farmer, E. Ott, and J. A. Yorke, "The dimension of chaotic attractors," Physica D: Nonlinear Phenomena, vol. 7, no. 1-3, pp. 153-180, 1983.

[9] A. Wolf, J. B. Swift, H. L. Swinney, and J. A. Vastano, "Determining Lyapunov exponents from a time series," Physica D: Nonlinear Phenomena, vol. 16, no. 3, pp. 285-317, 1985.

[10] A. M. Fraser and H. L. Swinney, "Independent coordinates for strange attractors from mutual information," Physical Review A, vol. 33, no. 2, pp. 1134-1140, 1986.

[11] C. G. Gilmore, "A new test for chaos," Journal of Economic Behavior and Organization, vol. 22, no. 2, pp. 209-237, 1993.

[12] J. D. Farmer and J. J. Sidorowich, "Predicting chaotic time series," Physical Review Letters, vol. 59, no. 8, pp. 845-848, 1987.

[13] M. Casdagli, "Nonlinear prediction of chaotic time series," Physica D: Nonlinear Phenomena, vol. 35, no. 3, pp. 335-356, 1989.

[14] M. Casdagli and A. Weigend, "Exploring the continuum between deterministic and stochastic modeling," in Forecasting the Future and Understanding the Past, A. Weigend and S. F. I. Gershenfeld, Eds., vol. 15 of Studies in the Sciences of Complexity, p. 993, Addison-Wesley, 1994.

[15] H. S. Kim, R. Eykholt, and J. D. Salas, "Delay time window and plateau onset of the correlation dimension for small data sets," Physical Review E, vol. 58, no. 5, pp. 5676-5682, 1998.

[16] H. S. Kim, R. Eykholt, and J. D. Salas, "Nonlinear dynamics, delay times, and embedding windows," Physica D: Nonlinear Phenomena, vol. 127, no. 1-2, pp. 48-60, 1999.

[17] M. Falanga and S. Petrosino, "Inferences on the source of longperiod seismicity at Campi Flegrei from polarization analysis and reconstruction of the asymptotic dynamics," Bulletin of Volcanology, vol. 74, no. 6, pp. 1537-1551, 2012.

[18] R. Benzi, A. Sutera, and A. Vulpiani, "The mechanism of stochastic resonance," Journal of Physics A. Mathematical and General, vol. 14, no. 11, pp. L453-L457, 1981.

[19] R. Benzi, G. Parisi, A. Sutera, and A. Vulpiani, "Stochastic resonance in climatic change," Tellus, vol. 34, pp. 10-16, 1982.
[20] C. Nicolis, "Stochastic aspects of climatic transitions-response to a periodic forcing," Tellus, vol. 34, pp. 1-9, 1982.

[21] I. Rodriguez-Iturbe, B. Febres De Power, M. B. Sharifi, and K. P. Georgakakos, "Chaos in rainfall," Water Resources Research, vol. 25, no. 7, pp. 1667-1675, 1989.

[22] B. P. Wilcox, M. S. Seyfried, and T. H. Matison, "Searching for chaotic dynamics in snowmelt runoff," Water Resources Research, vol. 27, no. 6, pp. 1005-1010, 1991.

[23] T. Sangoyomi, Climatic variability and dynamics of Great Salt Lake hydrology [Ph.D. thesis], Utah State University, Logan, Utah, USA, 1993.

[24] T. B. Sangoyomi, U. Lall, and H. D. I. Abarbanel, "Nonlinear dynamics of the Great Salt Lake: dimension estimation," Water Resources Research, vol. 32, no. 1, pp. 149-159, 1996.

[25] G. D. Jeong and A. R. Rao, "Chaos characteristics of tree ring series," Journal of Hydrology, vol. 182, no. 1-4, pp. 239-257, 1996.

[26] I. Rodriguez-Iturbe, D. Entekhabi, and R. L. Bras, "Nonlinear dynamics of soil moisture at climate scales, 1 . Stochastic analysis," Water Resources Research, vol. 27, no. 8, pp. 1899-1906, 1991.

[27] H. S. Kim, Y. N. Yoon, J. H. Kim, and J. H. Kim, "Searching for strange attractor in wastewater flow," Stochastic Environmental Research and Risk Assessment, vol. 15, no. 5, pp. 399-413, 2001.

[28] J. H. Ahn and H. S. Kim, "Nonlinear modeling of elnino/Southern osciilation index," Journal of Hydrologic Engineering, vol. 10, no. 1, pp. 8-15, 2005.

[29] H. S. Kim, K. H. Lee, M. S. Kyoung, B. Sivakumar, and E. T. Lee, "Measuring nonlinear dependence in hydrologic time series," Stochastic Environmental Research and Risk Assessment, vol. 23, no. 7, pp. 907-916, 2009.

[30] B. Sivakumar, F. M. Woldemeskel, and C. E. Puente, "Nonlinear analysis of rainfall variability in Australia," Stochastic Environmental Research and Risk Assessment, vol. 28, no. 1, pp. 17-27, 2014.

[31] S. Kim, H. Noh, N. Kang et al., "Noise reduction analysis of radar rainfall using chaotic dynamics and filtering techniques," Advances in Meteorology, vol. 2014, Article ID 517571, 10 pages, 2014.

[32] J. D. Salas, H. S. Kim, R. Eykholt, P. Burlando, and T. R. Green, "Aggregation and sampling in deterministic chaos: implications for chaos identification in hydrological processes," Nonlinear Processes in Geophysics, vol. 12, no. 4, pp. 557-567, 2005.

[33] C. Karamperidou, V. Engel, U. Lall, E. Stabenau, and T. J. Smith III, "Implications of multi-scale sea level and climate variability for coastal resources: a case study for south Florida and Everglades National Park, USA," Regional Environmental Change, vol. 13, no. 1, pp. 91-100, 2013.

[34] C. Chui, An Introduction to Wavelets, Wavelet Analysis and Its Application, vol. 1, Elsevier, New York, NY, USA, 1992.

[35] C. Torrence and G. P. Compo, "A Practical Guide to Wavelet Analysis," Bulletin of the American Meteorological Society, vol. 79, no. 1, pp. 61-78, 1998.

[36] C. Bishop, Neural Networks for Pattern Recognition, Clarendon Press, Oxford, UK, 2000.

[37] P. Picton, Neural Networks, Palgrave, Basingstoke, UK, 2nd edition, 2000.

[38] W. Hsieh, Machine Learning Methods in the Environmental Sciences, Cambridge University Press, Cambridge, UK, 2009.

[39] S. Haupt, A. Pasini, and C. Marzban, Artificial Intelligence Methods in the Environmental Sciences, Springer, Berlin, Germany, 2009. 
[40] R. J. Kuligowski and A. P. Barros, "Localized precipitation forecasts from a numerical weather prediction model using artificial neural networks," Weather and Forecasting, vol. 13, no. 4, pp. 1194-1204, 1998.

[41] Yuval and W. W. Hsieh, "An adaptive nonlinear MOS scheme for precipitation forecasts using neural networks," Weather Forecasting, vol. 18, no. 2, pp. 303-310, 2003.

[42] B. Sivakumar, K.-K. Phoon, S.-Y. Liong, and C.-Y. Liaw, "A systematic approach to noise reduction in chaotic hydrological time series," Journal of Hydrology, vol. 219, no. 3-4, pp. 103-135, 1999.

[43] A. Hyvärinen, J. Karhunen, and E. Oja, Independent Component Analysis, John Wiley \& Sons, New York, NY, USA, 2001.

[44] A. Hyvärinen and P. Pajunen, "Nonlinear independent component analysis: existence and uniqueness results," Neural Networks, vol. 12, no. 3, pp. 429-439, 1999.

[45] J. Basak, A. Sudarshan, D. Trivedi, and M. S. Santhanam, "Weather data mining using independent component analysis," The Journal of Machine Learning Research, vol. 5, pp. 239-253, 2003/04.

[46] P. Capuano, E. De Lauro, S. De Martino, and M. Falanga, "Water-level oscillations in the Adriatic Sea as coherent selfoscillations inferred by independent component analysis," Progress in Oceanography, vol. 91, no. 4, pp. 447-460, 2011.

[47] A. Ciaramella, E. De Lauro, S. De Martino, B. Di Lieto, M. Falanga, and R. Tagliaferri, "Characterization of Strombolian events by using independent component analysis," Nonlinear Processes in Geophysics, vol. 11, no. 4, pp. 453-461, 2004.

[48] E. de Lauro, S. de Martino, M. Falanga, and M. Palo, "Decomposition of high-frequency seismic wavefield of the Strombolianlike explosions at Erebus volcano by independent component analysis," Geophysical Journal International, vol. 177, no. 3, pp. 1399-1406, 2009.

[49] M. S. Karoui, Y. Deville, S. Hosseini, A. Ouamri, and D. Ducrot, "Improvement of remote sensing multispectral image classification by using Independent Component Analysis," in Proceedings of the 1st Workshop on Hyperspectral Image and Signal Processing: Evolution in Remote Sensing (WHISPERS '09), pp. 1-4, IEEE, August 2009.

[50] L. Chen and C. Lu, "An improved independent component analysis algorithm based on artificial immune system," International Journal of Machine Learning and Computing, vol. 3, no. 1, pp. 9397, 2013.

[51] J. Holzfuss and G. Mayer-Kress, "An approach to errorestimation in the application of dimension algorithms," in Dimensions and Entropies in Chaotic Systems, G. Mayer-Kress, Ed., vol. 32, pp. 114-122, Springer, New York, NY, USA, 1986.

[52] K. E. Graf and T. Elbert, "Dimensional analysis of the waking EEG," in Chaos in Brain Function, E. Basar, Ed., pp. 135-152, Springer, Berlin, Germany, 1990.

[53] A. A. Tsonis and J. B. Elsner, "The weather attractor over very short time scales," Nature, vol. 333, no. 6173, pp. 545-547, 1988.

[54] J. Fourier, Théorie Analytique de la Chaleur, Firmin Didot Père et Fils, Paris, France, 1822, (French). 

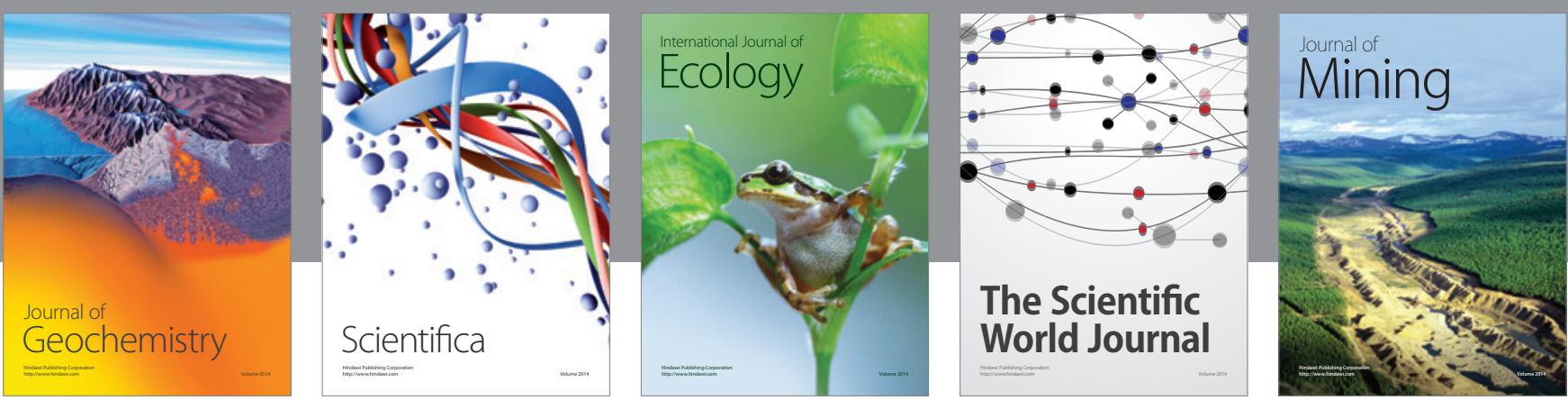

The Scientific World Journal
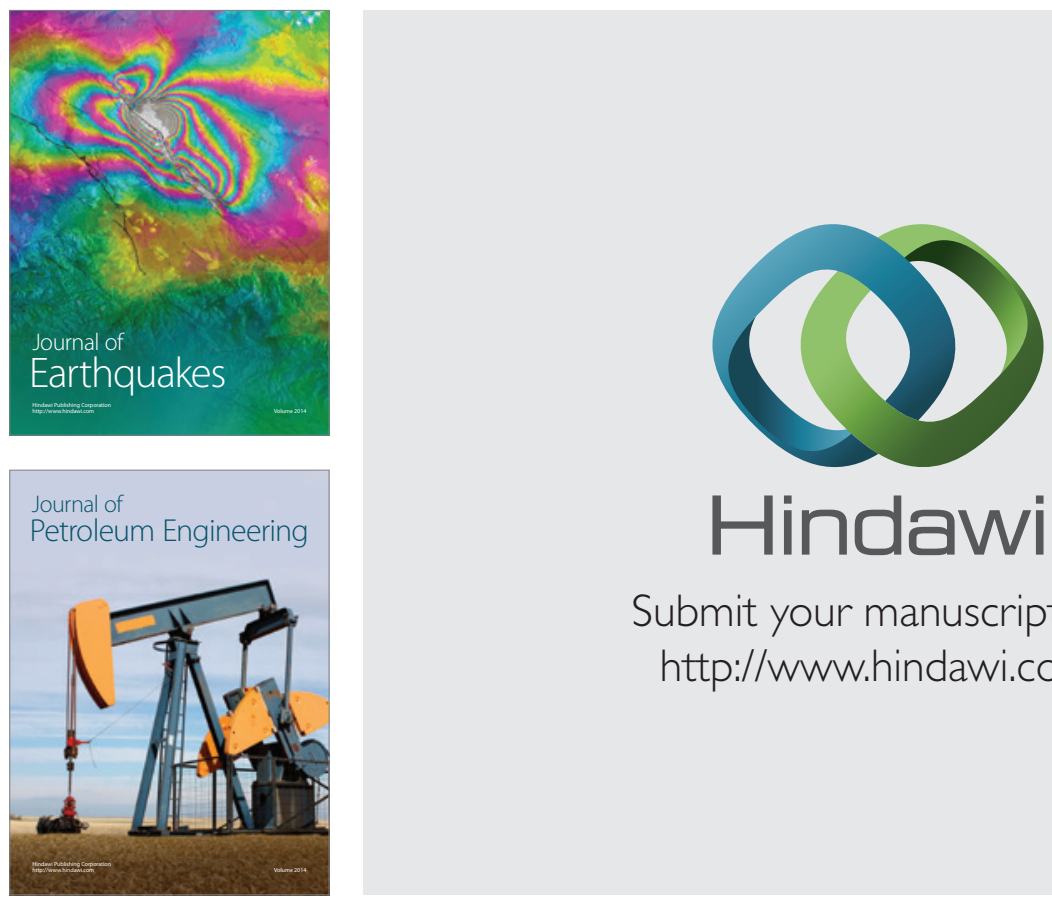

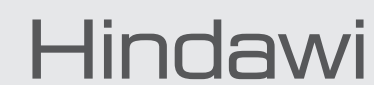

Submit your manuscripts at

http://www.hindawi.com
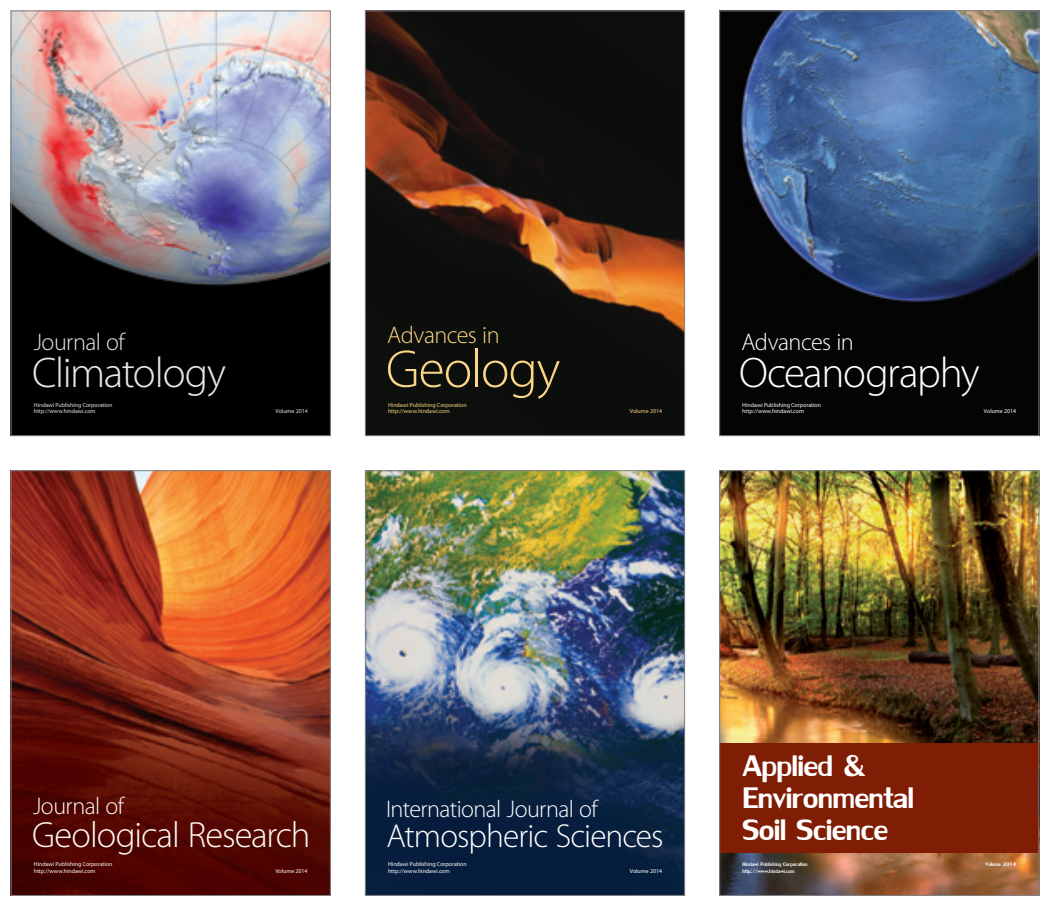
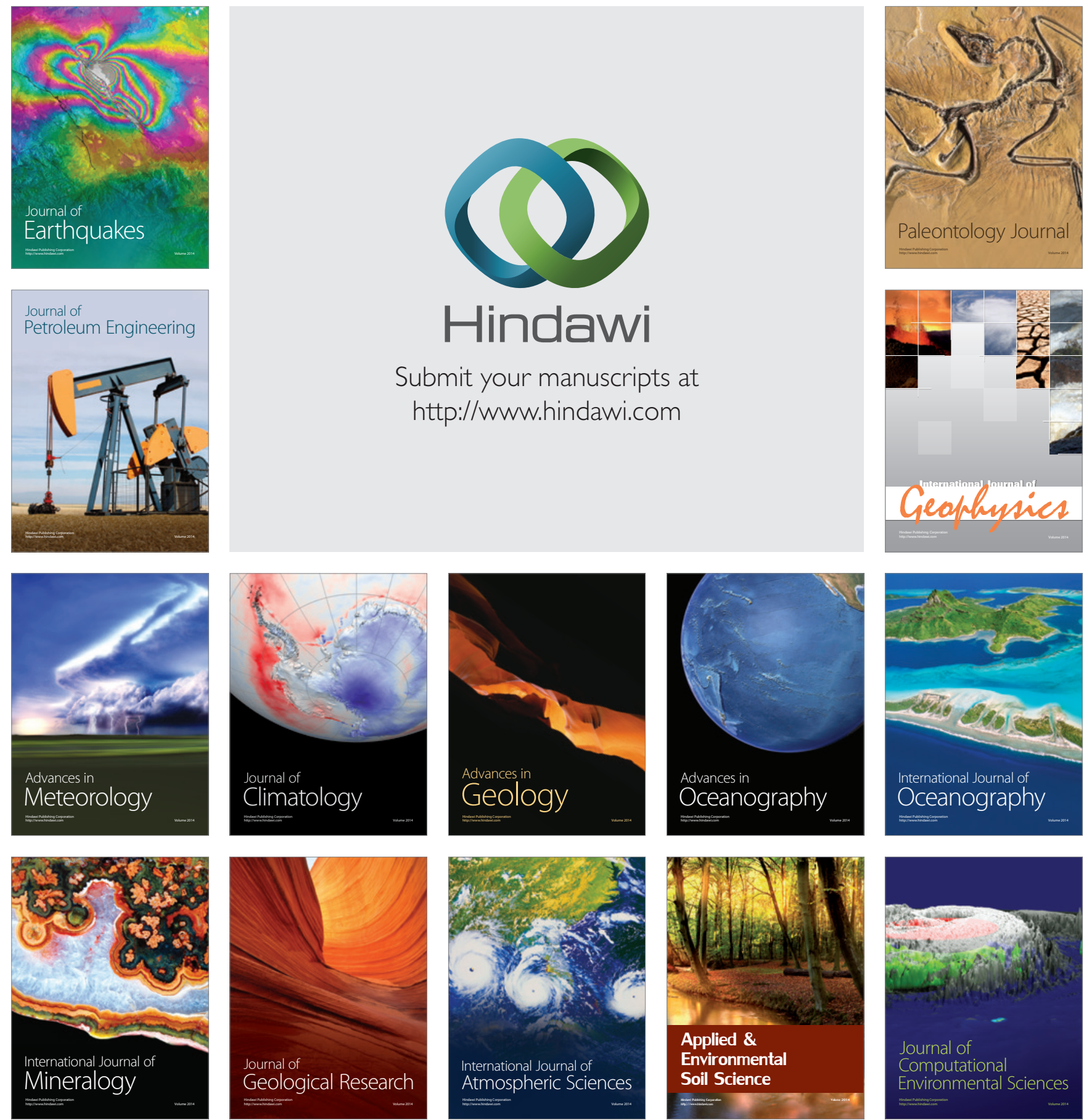